\title{
Belphégor
}

\section{Alexandre Dumas : mal écrire, bien écrire}

\section{Charles Grivel}

\section{(2) OpenEdition}

\section{Journals}

Electronic version

URL: https://journals.openedition.org/belphegor/1374

DOI: 10.4000/belphegor.1374

ISSN: 1499-7185

\section{Publisher}

LPCM

\section{Electronic reference}

Charles Grivel, "Alexandre Dumas : mal écrire, bien écrire", Belphégor [Online], 16-1 | 2018, Online since 17 July 2018, connection on 19 October 2021. URL: http://journals.openedition.org/belphegor/1374 ; DOI: https://doi.org/10.4000/belphegor.1374

This text was automatically generated on 19 October 2021.

\section{(c) (i) (9)}

Belphégor est mis à disposition selon les termes de la Licence Creative Commons Attribution - Pas d'Utilisation Commerciale - Pas de Modification 4.0 International. 


\title{
Alexandre Dumas : mal écrire, bien écrire
}

\author{
Charles Grivel
}

\section{REFERENCES}

Parution originale dans Fernande Bassan et Claude Schopp (dir.), Les Trois Mousquetaires, Le Comte de Monte Cristo, cent cinquante ans après, Marly-Le-Roy, Éditions Champflour, 1995.

1 Il y a un cas Dumas, nous ne nous réunirions pas, du reste, en sa compagnie sans cela. Ce cas est singulier : il n'a jamais été question de rendre tout simplement hommage à l'archi-célèbre auteur. Car les choses sont ainsi que toute la critique - non ses lecteurs est critique envers lui, qu'elle soit ennemie ou amie, d'autrefois à aujourd'hui. Cette attitude dépasse le racisme ordinaire: comment se peut-il faire qu'un noir, et de surcroît républicain ou s'efforçant d'en convaincre, ou pis : monarchiste de gauche, se hisse au pinacle de la littérature nationale? C'est ce qui paraît intolérable au contemporain. Comment un conteur populaire pourrait-il prétendre aux fastes institutionnels et universitaires réservés aux bons auteurs?

2 Cela continue de troubler le bon goût d'aujourd'hui. Or, l'opiniâtreté, l'exclusivité et aussi la violence d'un tel jugement dépassent la norme : bien des auteurs populaires à succès peuvent attirer sur eux, à un titre ou à un autre, la vindicte sociale, beaucoup parmi eux ont laissé une oeuvre à la fois conforme et non conforme, mais Dumas focalise mieux qu'un autre les regards indignés ou méfiants de toutes les factions. Je me demande pourquoi. Je me demande sur quel terrain durable repose l'opprobre ou alors le malentendu. Je crois qu'il y a anguille sous roche. Mon soupçon est conforté par le fait que la rancœur critique s'en prend, presque systématiquement, au style de l'écrivain, au bâclage dont il fait montre, comme à l'odeur de fabrique qui distinguerait ses écrits : Dumas le grand copieur, Dumas le falsificateur, on ne se fait pas faute de le dire! 
De fait, Alexandre Dumas - le nom, le mythe, la star - offre un immense terrain à l'expression vindicative ou revendicatrice des commentateurs. En voici quelques exemples. Nous verrons plus tard de quoi ils procèdent. Portrait-robot avec incise négative tiré d'un volume intitulé Alexandre Dumas à Tunis: hâblerie "incorrigible ", ingénuité et "bon-garçonnisme désarmants ", sympathie, sa vie est un défi à toutes les vraisemblances, son œuvre aussi, sa bonté [surtout sa bonté] sa prodigieuse fertilité, sa virtuosité, sa verve, bien sûr, tout ce qu'il a signé n'est pas de lui et l'histoire qu'il écrit, peut-être pas vraie, a pourtant la vertu d'avoir de la vie..., et ainsi de suite ${ }^{1}$.

4 Ce Dumas-là ressemble furieusement à ce que l'on attend de lui : parodie de style de feuilletoniste "à la Dumas ", supposé être sa facture-type, avec clin d'œil au lecteur, sous forme de poème: "DE CAPE ET D'ÉPÉE. Dans une sombre demeure dans une sombre villa vivait un sombre personnage. Il riait sombrement et fomentait d'obscurs projets. Sa silhouette opaque se découpait en ombre chinoise à la fenêtre lorsqu'il contemplait la nuit, les redoutables pensées niellaient l'éclat de ses yeux charbonneux. Il proférait entre les caries de sa denture d'inquiétantes menaces. Il portait le deuil et s'adonnait à de ténébreux souvenirs. Des rides funestes parcouraient sa face ombrageuse, et sa cape crépusculaire frémissait de reflets sataniques. C'était le dernier mousquetaire de la Compagnie de Tréville $»^{2}$.

5 Côté roman policier, maintenant: dans une petite ville sidérurgique de l'Est, un jeune écrivain désargenté accepte d'écrire l'autobiographie d'une idole de la chanson argument du commanditaire - véreux, comme il se doit - pour convaincre celui qui a encore des scrupules et voudrait au moins ne pas engager son nom dans une telle entreprise : «Comme si Jules Verne n'avait pas écrit la moitié des livres de Dumas! $»^{3}$. L'affirmation a beau être grosse, elle trouve pourtant son auditeur, et avec lui son lecteur, sans doute crédule!

6 Voici maintenant un article de presse consacré à l'informatisation du savoir, et une caricature illustrant démonstrativement cet article. Un enfant est assis devant le clavier. L'écran affiche: ALEXANDRE DUMAS, LES TROIS MOUSQUETAIRES. L'amorce d'un texte suit, sur deux colonnes. Question de l'enfant dans une bulle: «Dis papa, c'était comment un livre?». Mine perplexe de l'adulte ${ }^{4}$, Le roman dumasien perdure, il a pourtant ici disparu, du moins son support - le livre-, dont tout indique - la caricature justement- qu'il n'est pas détachable.

7 Tant d'indices -chacun aura complété le dossier- rendent songeur. Que Dumas soit accommodé à toutes les sauces et qu'il fasse figure d'homme à tout faire des lettres n'est pas le pire, mais qu'il serve à alimenter un déni d'écriture : la sienne, d'abord !

Dumas a fait face à une campagne de dénigrement d'une rare ampleur, de durée remarquable, de singulière nature. Les appréciations qui accompagnent la parution de ses ouvrages, comme les faits et gestes de l'homme public relèvent, autrefois comme aujourd'hui, de racisme ordinaire. Le crime d'opinion est apparemment bien moins grand que le crime de couleur, aussi malsonnant que cela puisse paraître, un siècle et demi après la parution de ses plus fameux ouvrages et en ce jour anniversaire. Même si, au fil des ans, les vieux partis-pris prennent souvent [mais pas toujours !] une forme plus rampante et plus discrète.

9 Ce qu'on appelle sa «bonté » [la « bonhomie »], sa « naïveté », cet amour qu'il aurait du tape-à-l'œil, du "clinquant», de l' «or», du luxe de mauvais aloi, ce goût caractéristique dont il ferait montre pour le tapage - Alexandre Dumas est en effet un 
être bruyant - cet appétit des femmes - on lui prête un grand nombre de maîtresses ${ }^{5}$-, contribuent à son discrédit et sont portés au compte de sa "négritude ». Celle-ci a beau avoir quelque chose de légendaire pour le parvenu de la monarchie de Juillet et pour ses lecteurs qui ne sont pas allés y regarder de si près, peu importe, c'est ainsi qu'il a lui-même voulu vivre le déni qui le frappe, et c'est ainsi aussi que ses détracteurs ont voulu le caractériser. Du reste, son œuvre lève à cet égard toute ambiguïté : Alexandre Dumas est bien un noir qui écrit ${ }^{6}$.

Voici maintenant le portrait de Dumas. Je puise à peu près au hasard, on s'en doute, pour constituer ce petit florilège :

Gautier en 1848: «Dumas est créole, à part toute allusion de peau » ${ }^{7}$. La restriction implique le renforcement.

Lectures pour tous, février 1930: "Dumas n'était ni fumeur ni priseur. Quel était le stimulant de son inspiration ? On ne le devinerait jamais... De la limonade gazeuse ! [...]. Enclin à la gloriole des titres, il usait d'un papier à lettres timbré d'une couronne de comte, sans doute en raison de son aïeul, gentilhomme français qui avait émigré aux Antilles [...]. Mais il n'en était que le descendant naturel, son père étant fils d'une esclave haïtienne [...]. Il recherchait les décorations et n'était jamais si heureux que les soirs de gala où il chamarrait son habit d'une guirlande d'ordres étincelants ». Un tel portrait parle un langage clair.

Paul Morand, préface au Vicomte de Bragelonne, à propos des cinq cents lignes de Maquet et des trente mots de l'écriture de Dumas, comme en atteste le manuscrit retrouvé d'un feuilleton: "Mais ces trente mots réveillent tout, effacent la documentation, imposent ce génie de féticheur africain qu'est celui de Dumas ${ }^{8}$, La précision tempère la louange. Claude Silvain note dans Humeurs et humour d'Alexandre Dumas [1993]: «Des excès de vie, c'est peu dire. Dumas père jouissait de tous les plaisirs, les femmes et la bonne chère. Mais sa troisième passion, l'écriture, dominait tout le reste ». Le comportement libertin du natif de Villers-Cotterêts proviendrait du château du lieu, de mauvaise réputation, car il aurait servi de cadre à des « orgies indescriptibles » où l'on soupait nu $[!]^{9}$. On voit où veut porter l'accent.

15 Daniel Zimmermann, dernier biographe de Dumas [le passage se réfère au mûrissement de ce qui donnera Henri III et sa cour] «Alexandre commence à entrevoir son SaintMégrin, jeune, beau, élégant, brave, insolent, à peine équivoque, son portrait craché [ !], «mignon » du roi, moins au sens de giton que favori ayant une relation de type filial avec un aîné au statut plus prestigieux que le sien, cela aussi Alexandre peut comprendre aisément. Il est en effet fort improbable qu'il se soit jamais reconnu de penchants homosexuels. Néanmoins l'androgyne, comme le travesti homme ou femme, si fréquent dans l'œuvre de son maître Shakespeare, sont des personnages qui le séduisent. Lui-même est très attiré par la beauté masculine, ce qui ne l'empêche nullement de jouer de la sienne avec les deux sexes. Par-dessus tout, il a le culte de la virilité, sans doute en référence au Général idéalisé $»^{10}$. On appréciera la valsehésitation. Au reste, en contrepartie [?], l'auteur insiste pesamment sur ce qu'il présente comme des côtés ridicules du grand homme, et ne se prive vraiment pas d'adresser force clins d'œil au lecteur à propos de sa plus mince aventure galante. Les passages qu'on vient de lire, révèlent une insistance par les incises, les implications, les formules à moitié formulées, les réticences, toute la texture du texte. S'agissant de décrier un personnage du calibre d'Alexandre Dumas, on comprend bien qu'il faut éviter les déclarations franchement intempestives. N'empêche, Dumas, comme très peu 
de ses confrères, fait les frais de l'idée préconçue et du parti pris - discret ou non - de ses critiques : pour délit sexuel ou délit de négritude, c'est tout un.

L'accent critique porte pourtant sur un autre point : l'écriture. On reproche à Dumas de trop écrire, de mal écrire et même de ne pas écrire ce qu'il publie. Il faut bien se rendre compte, ici, que l'auteur des Trois Mousquetaires " par Maquet interposé » a droit, si l'on peut dire, à un régime de faveur. Beaucoup de ses confrères se sont assuré les services d'un « nègre » ou d'un " secrétaire », beaucoup ont puisé le meilleur de leur inspiration dans le texte d'autrui, et quoi qu'il en soit nous savons depuis longtemps - depuis Flaubert au moins - que le poncif circule et que la bibliothèque est une immense carrière où chaque écrivain vient tailler sa matière. Le déni frappe donc à la fois l'homme et l'œuvre: l'homme certainement, parce qu'il ne prend pas soin de dissimuler ses façons de faire, bien au contraire; l'œuvre, parce que l'authenticité mais aussi la propriété et l'identité - ne la cautionnent pas. Or, un fait particulier marque la carrière de Dumas et explique, à mon sens, pour une bonne part, l'éclosion de la suspicion: le fait de se situer sur la ligne de clivage entre Ancien Régime et Nouveau Régime, en littérature s'entend. Ses œuvres, par leur côté héroïque, participent de la mise en place de l'esthétique romantique. Ses œuvres se plient aux obligations qui sont celles de la littérature «industrielle» - obligations presque immédiatement repérées par Sainte-Beuve. En œuvrant de double registre, en participant à deux batailles, il ne pouvait que décevoir ou heurter. Dumas incarne, il l'a pour une bonne part voulu, un type nouveau de littérateur, c'est un faiseur professionnel et non pas un inspiré, il ne souscrit pas à l'esthétique officielle, sans pourtant en rabattre sur ses prétentions dans la course aux honneurs.

Or, cette réaction n'aurait pas été si tenace et si violente, si l'écrivain n'avait pas suivi un programme de publication exorbitant, « universel» comme il disait. Ce qui fait scandale chez Dumas, c'est qu'il s'identifie à ce qu'il écrit. Or, et c'est ce qui fait de son œuvre un mélange détonnant, la visée de l'homme aux quatre cents romans et aux cinq cents enfants - chiffres qui ont été articulés -, c'est qu'il prend place au centre d'une toile qui réunit d'infinies cohortes de personnages et de figurants : «J'ai pris ce livre,[il s'agit des Mémoires] comme un cadre immense pour vous y faire entrer tous, frères et sœurs en art, pères ou enfants du siècle, grands esprits, corps charmants, [...] vous qui m'avez aimé, et que j'ai aimés [...] ; vous qui m'êtes restés inconnus ; vous-mêmes qui m'avez haï ! [...] En m'adjoignant à vous, [...] je deviens un des anneaux de la chaîne d'or qui relie le passé à l'avenir. Non, ce ne sont pas mes Mémoires que j'écris [...], ce sont les Mémoires de la France $»^{11}$.

18 L'auteur Dumas se représente parmi ses créatures, comme l'une de ses propres créatures. Trônant et statufié, mais mêlé à elles. C'est ainsi qu'on le rencontre dans les gravures qui ornent l'édition illustrée d'Histoire de mes bêtes ou dans la caricature, sur le dessin de Paul Destez par exemple ${ }^{12}$, La plus belle allégorie est pourtant celle qui figure gravée dans la pierre sur les façades du château d'If en sa propriété de Monte-Cristo : morceau par morceau et au vu du lecteur, les titres de ses ouvrages constituent ensemble sa demeure. Même optique dans une supplique qu'Alexandre Dumas adresse à "Napoléon le petit» dans le but de faire lever un arrêt de censure. Il se compare à Hugo et à Lamartine, plus dignes, certes, mais moins populaires : «J'ai écrit et publié douze cents volumes [...]. Traduits dans toutes les langues, ils ont été aussi loin que la vapeur a pu les porter». Et il ajoute, dans un louable esprit de conciliation, mais non dépourvu d'arrière-pensée, que tous ces volumes d'un simple « vulgarisateur » - c'est le 
mot qu'à dessein il emploie - conviennent à l'ouvrier autant qu'à la jeune fille, au républicain endurci aussi bien qu'à l'aristocrate effarouché13. L'écrit de Dumas fait masse. Il se répand, il atteint en étendue et en profondeur toutes les couches. La visée dumasienne est l'universalité et l'hôte de Monte-Cristo se donne pour tâche d'écrire tous les livres, d'écrire à tous, toutes opinions confondues, tout ce qu'ils désirent lire. La littérature universelle répond en lui à l'universelle demande.

19 L'auteur de tant de livres donne dans la fabrique; son tort est de revendiquer à ce niveau-là, et bruyamment, la reconnaissance d'un pouvoir qu'il concurrence. L'Empire érigé grâce au suffrage universel ne pouvait pas laisser s'exprimer la voix de tous, la voix pour tous. Dumas, dans sa littérature, se place instinctivement dans la ligne d'une culture politique impossible à assumer. Ce qu'il propose - quelque chose comme la volonté générale du Peuple mise en fiction et fondue en littérature - est, vu de l'époque, l'inadmissible par excellence. Pourtant l'époque invente une "propriété littéraire » fondatrice du négoce auquel Dumas - et les plus malins de ses confrères - se livrent, sans pourtant clairement l'admettre. D'un côté, elle fait du livre un produit, de l'autre, elle lui recommande d'échapper aux lois de la production. D'un côté, ses directeurs de journaux s'efforcent d'argumenter les ventes; de l'autre, ses critiques s'acharnent à dénoncer précisément celle-ci. Voici donc l'auteur pris entre deux feux, particulièrement s'il désire être vainqueur sur les deux tableaux.

L'attitude de Gustave Planche, pontife de la critique des années 1830, est à cet égard particulièrement instructive. Voici ce qu'il écrit de Teresa de Dumas, drame représenté pour la première fois à l'Opéra-Comique, le 6 février 1832: « Il y a des hors-d'œuvre, des déclamations, de l'emphase, il n'y a pas une tirade précise et pleine. En un mot, la pièce n'est pas écrite, il n'y a pas de style. La méditation et le soin ont manqué. Déjà Henri III et Antony avaient mérité le même reproche. Dans Teresa, la négligence littéraire est plus flagrante encore. Que M. Dumas y prenne garde, le style seul fait la durée des œuvres poétiques ». A propos d'Angèle [28.12.1833], le verdict est encore moins mesuré, Planche indiquant que l'auteur viole les plus vulgaires bienséances, qu'il possède des idées, mais se montre incapable de les développer, que le sublime de la pièce, apparemment existant, est dû au hasard. Et il conclut : " Non, le succès ne justifie pas l'œuvre. Non, ce n'est pas là de la poésie pure et vraie. » Aggravation des reproches à la sortie de Don Juan de Marana [1836], considéré comme un entassement de scènes avec d'innombrables plagiats: "C'est un garde-meuble ", un "mémorial », dit Planche en veine de métaphores. "Ces plagiats», ajoute-t-il, "Monsieur Dumas n'a pas eu le courage ou l'habileté de les frapper à son effigie ; toutes les scènes, tous les incidents, toutes les images et toutes les rimes aussi [sic] étant plagiés, point n'était nécessaire de nous les représenter ! Conclusion: «Tout l'avenir de ce drame fantastique repose sur l'habileté du machiniste ${ }^{14}$, Même son de cloche chez Théophile Gautier dont la plume, pour être plus circonspecte, n'en est pas moins acérée. En date du $1^{\mathrm{er}}$ janvier 1838, celui-ci fait précéder les quelques mots qu'il consacre au Caligula de Dumas dans sa rubrique d'un long réquisitoire contre la collaboration au théâtre: "C'est une décadence complète; la manufacture envahit tout; une pièce se fabrique absolument comme un habit: l'un des collaborateurs prend la mesure de l'acteur, l'autre coupe l'étoffe et le troisième assemble les morceaux; l'étude du cœur humain, le style, la langue tout cela est regardé comme rien ». La collaboration pour une œuvre de l'intelligence, est quelque chose d'incompréhensible, et dont il ne peut résulter que des produits hybrides et monstrueux. Ce qui, dans le contexte, ne manque pas de piquant, surtout si l'on met en regard le fait que les critiques ne trouvent à louer dans la pièce 
dont ils rendent compte, hormis ses «beaux vers bien rythmés de saveur tout antique ", que la présence des chevaux sur la scène que l'auteur eu le grand mérite, à ses yeux, de savoir imposer à la direction du théâtre qui accueillait la pièce ${ }^{15}$.

Certains critiques ont reproché à Dumas ses collaborateurs, notamment Mirecourt et Clémence Badère - femme de lettres éconduite au Mousquetaire, quelques années plus tard - ; le fond de l'argumentation haineuse est le même et les formulations dictées par l'envie. Cette femme-auteur suppose qu'Alexandre Dumas s'acharne à faire en sorte que ses écrits à elle ne paraissent point. Il s'agirait là d'une vindicte de despote. Cet ogre des lettres avalerait tout ce qui l'approche, ce soleil de la littérature s'ingénierait à briller seul, mais d'une gloire d'emprunt, des soixante "rayons" que lui fourbissent ses collaborateurs : «Non, monsieur Dumas, non, vous ne faites pas soixante volumes par an ; il n'y a pas d'imagination, si féconde qu'elle soit, qui puisse produire cela ». Dumas est un "arrangeur»; il signe ce qu'il n'écrit pas; la preuve en est qu'un texte de lui recopié de sa main et signé de mon nom - je suis la démonstration de l'accusatrice essuie le refus d'un censeur, pourtant fervent admirateur du grand homme, appelé comme arbitre. Elle conclut : « Dumas n'est pas Dumas et moi je suis moi-même ! ${ }^{16}$.

21 Pour en terminer avec le témoignage des contemporains, examinons l'argumentation de Sainte-Beuve, dont on attend bien sûr qu'il s'entoure d'un peu plus de précautions. Mais prince de la critique ou pas, c'est au même endroit que le bât blesse. C'est ainsi qu'on le voit tenter de dépeindre, dans son feuilleton du premier juillet 1843, une situation littéraire évidemment calamiteuse : depuis la vague byronienne qui a déferlé sur le continent, explique-t-il, «la plupart des gens de talent en vers et en prose sont fats, c'est-à-dire affichent ce qu'ils n'ont pas, affectent ce qu'ils ne sont pas, même les critiques [sic] [...]. La fatuité combinée à la cupidité, à l'industrialisme, au besoin d'exploiter fructueusement les mauvais penchants du public, a produit, dans les œuvres d'imagination et dans le roman, un raffinement d'immoralité et de dépravation qui devient un fait de plus en plus quotidien et caractéristique, une plaie ignoble et livide [?] qui chaque matin s'étend. ».

22 Ce diagnostic sociologique que le sérieux supposé du médecin positif dissimule Sainte-Beuve crédite le phénomène d'un " fond de sanie »-, on le trouve, en 1839 déjà, dans son célèbre "De la Littérature industrielle ", relié à ce qu'il appelle "le démon de la propriété littéraire».

23 J'ai montré plus haut que ce n'était pas un hasard: le marché exige la fabrique, les auteurs ne sont plus des prométhées, quoiqu'on exige d'eux pourtant qu'ils endossent la tenue de l'original [voire du grand barde] pour être reconnus à leur place. SainteBeuve, qui ne cite pas Dumas, s'en prend donc aux ravages que le système industriel fait encourir dans les journaux à la littérature d'imagination particulièrement: "Les feuilletons se distendent indéfiniment, l'élasticité des phrases a dû prêter, et l'on a redoublé de vains mots, de descriptions oiseuses, d'épithètes redondantes : le style s'est étiré dans tous ses fils comme les étoffes trop tendues ».

24 «Il y a des auteurs - suivez mon regard! - qui n'écrivent plus leurs romans de feuilletons qu'en dialogue, parce qu'à chaque phrase, et quelquefois à chaque mot, il y a du blanc, et que l'on gagne une ligne.». Le 15 septembre 1840, dans le cadre d'une chronique qu'il consacre à Eugène Sue, le critique reviendra sur sa thèse alarmiste : "une société ", dit-il. "a la littérature qu'elle mérite ", et c'est à ce propos qu'il évoquera Dumas: "S'il devient banal de redire que la littérature est l'expression de la 
société, il n'est pas moins vrai d'ajouter que la société aussi se fait l'expression volontiers et la traduction de la littérature.

Tout auteur tant soit peu influent et à la mode crée un monde qui le copie, qui le continue, et ce point-là, excité qu'il est et comme piqué d'honneur, se développe à l'envi et se met à ressembler davantage. Lord Byron - décidément coupable ! - a eu depuis longtemps ce rôle d'influence sur les hommes [...]. Depuis ç'a été le tour des femmes; l'émulation les a prises de lutter au sérieux avec les types, à peine apparus, d'Indiana ou de Lélia. Je me rappelle avoir été témoin certain soir et dans un hôtel de la meilleure compagnie d'un drame domestique réel très imprévu, et qui justifiait tous ceux de Dumas, etc., etc." ${ }^{17}$. Je trouve curieux que notre auteur soit mentionné à ce propos.

On aurait tort de penser que ces plaintes ont cessé avec le dix-neuvième siècle et que Dumas a été lavé du soupçon d'être le responsable en chef de la pire des tares littéraires. Je ne prendrai que trois exemples, récents mais augustes! Gérard Genette, théoricien de la littérature dans Seuils ${ }^{18}$ - remarquable par ailleurs - consacré à l'appareil de présentation du texte [au "paratexte»] en vient à discuter du plagiat. Concernant ce qu'il range dans la catégorie du " plagiat consenti » " par le plagié, bien sûr, et selon rétribution ", voici ce que le lecteur peut lire à propos du seul exemple proposé : "Chacun sait qu'Alexandre Dumas se faisait fréquemment aider [entre autres] par un professionnel nommé Auguste Maquet : c'est ici le porte-plume, inverse du prête-nom ».

Le romancier Arturo Pérez-Reverte, dans un ouvrage à succès fantaisiste, Le Club Dumas $^{19}$, s'ingénie, sur près de quatre cents pages, à démontrer - je veux bien que cela soit pour la bonne cause, puisque le récit présente quelque chose comme une morale pro-dumasienne - que notre auteur n'est pas le vrai père des Trois Mousquetaires. Ce « club Dumas » réunit du beau monde, dont le professeur de sémiologie de l'université de Bologne, Umberto Eco [à qui nous allons avoir à faire dans un instant], se serait donné pour tâche de préserver la mémoire d'Alexandre Dumas en ne rendant pas publique l'existence d'un manuscrit de son collaborateur Maquet constituant la preuve de l'imposture. Rappelons qu'il n'existe pas de manuscrit intégral des Trois Mousquetaires de la main de Dumas, qu'il existe, par contre, un manuscrit partiel rédigé par son collaborateur - soixante-trois pages de l'édition des Classiques Garnier - et que ces faits sont connus, depuis l'édition de 1956 en question par Charles Samaran.

Mentionnons l'essai très performant d'Umberto Eco, De Superman au surhomme $e^{20}$ : thèses fracassantes auxquelles la presse a fait très largement écho - sans jeu de mot: la postulation du surhomme de sinistre mémoire provient moins du Zarathoustra nietzschéen que de Monte-Cristo, héros plus que douteux, lancé sur le marché des mythes par Dumas lui-même [la dénonciation se trouve chez Gramsci] ${ }^{21}$, roman le plus célèbre de Dumas est selon lui un mauvais roman [«Le Comte de Monte-Cristo est sans aucun doute l'un des romans les plus passionnants qui aient jamais été écrits, c'est aussi l'un des romans les plus mal écrits de tous les temps et de toutes les littératures»), faux ou pas, décousu mais homérique, hors du champ de l'art et incompatible avec celui de la littérature. Selon lui c'est un livre manipulateur et «qui nous parle d'une certaine manière de la physiologie de nos tripes ", plutôt du côté des mythes Bororo. Umberto Eco est dur, très dur, et injuste, poussé par la rigueur qu'il croît reconnaître à la pensée la plus spéculative. Mais il faut souscrire à ce qu'il dit du Vicomte de Bragelonne, «c'est un roman-feuilleton qui semble vaincu par les dures nécessités de son propre marché ", 
mais de cela il ne s'ensuit pas qu'il n'ait pas été écrit, lui et ces textes qu'Alexandre prend la liberté de signer, pour innombrables qu'ils soient. Ecrit est le mot, le mot du pouvoir et celui de l'énigme.

Un théoricien, un romancier, un professeur, chacun des plus notoires dans sa partie, reprennent donc aujourd'hui à leur compte, par la bande ou sans détour, la vieille affirmation. Il m'a semblé que le fait, symptomatique de notre façon de pratiquer la littérature et la lecture, devait être relevé.

Prenons du recul et tentons l'explication. Je ne ferai qu'esquisser, on s'en doute. D'abord, Alexandre Dumas touche à l'Histoire. Il a compris plus vite que d'autres que l'Histoire est constituée par une suite ininterrompue de récits, qu'elle est entassement d'écriture, que l'écrire, cette Histoire, c'est en proposer une nouvelle version, ajouter à l'univers des discours, grimper d'un degré sur l'échelle mobile du temps, hausser la perspective, élargir, appréhender, mais toujours à partir de ce que la main est capable d'en tracer. Ecrire, c'est récrire, et la base est compilatoire. On suppose des faits, on désigne des événements, des personnages agissent, des batailles se font, des empires, des révolutions, on invente le roman historique.

Dumas en devient peut-être en France le principal promoteur, il place même l'Histoire sur les planches, son théâtre sera, comme on sait, et de plus en plus jusqu'à sa chute, celui de la reconstitution historique : en un mot, d'une péripétie réaliste, Alexandre fait une fiction. Ce faisant, il touche au principe même d'identité nationale : on supporte un Britannicus ou un Jugurtha de pacotille, mais un Louis, un Philippe inspire de toutes autres passions. L'Histoire est donc, mais bien plus scandaleusement vu l'investissement socio-politique qu'elle implique, matière littéraire. Comme le dit Dumas, l'Histoire est ce que j'en raconte. La thèse était moderne, plus que moderne ! et provocatrice.

Deuxièmement, Alexandre Dumas « attente à la littérature ». Dans un monde qui a mis en avant la personnalité de l'auteur, son originalité, sa sincérité et qui continue de le faire en dépit de la commercialisation galopante qui gagne, sous la monarchie de Juillet, la sphère culturelle. Dumas se présente [sans s'embarrasser de scrupules et sans le taire, et cela aussi le distingue de ses confrères] comme un "metteur en œuvre $»^{22}$, un arrangeur de la matière à écrire fournie par d'autres. La littérature devient à ses yeux et sous sa plume, de la littérature populaire. Non pas qu'il faille entendre par là qu'elle est de moins bonne qualité, mais qu'elle répond désormais aux critères de rapidité et de généralité : c'est une littérature vite faite, vite lue, vite produite et vite proposée à son consommateur; elle circule par conséquent, elle s'étend, se distribue, il y en a bientôt tous les jours, en tous lieux, et pour tous les goûts.

Cette littérature-lectures pour tous se fonde sur l'intérêt, le dialogue et la visualisation. La règle des genres n'a plus guère prise sur elle; elle tend, d'abord par le biais de l'Histoire, puis par celui du fait-divers, à devenir partie intégrante du tissu quotidien. Cette fictionnalisation n'est plus apte à remplir une fonction cathartique, ce n'est plus un mana, c'est simplement l'exhaussement de l'ordinaire à sa plus forte puissance, devenue fiction.

On peut certes avoir le regard braqué sur les galopades et les prouesses des récits dumasiens, et il n'en manque pas! mais on doit aussi redescendre de ces hauteurs et se rendre compte que le lieu où ces fresques opèrent est le salon, le foyer, le café, la rue. Avec Dumas, le roman descend du ciel sur terre, il opère sa mutation bourgeoise, il est 
devenu une façon de lire son journal et de vivre pareillement à tout ce qui s'y trouve écrit.

Voici donc tirées par notre auteur les conséquences liées à la double révolution politique et industrielle que connaît son époque : dans la société démocratique dont les bases sont maintenant posées, chacun peut accéder au statut de lecteur, et sa faim, il va falloir la nourrir. Chacun même peut accéder à celui d'auteur, il lui suffit de développer un certain savoir-faire pour cela, d'accepter de remanier, de se considérer non plus comme un inspiré, mais comme un professionnel.

L'Histoire, la Littérature, la Personne et le Moi sont les quatre grands piliers du Temple. Nouveau Samson, Alexandre Dumas les aura fait vaciller un instant pour notre entière satisfaction.

\section{NOTES}

1. Impressions de voyage, présentées, annotées et illustrées par Moncef Charfeddine, Tunis, 1982. Nous avons affaire ici à un extrait du Véloce. La couverture représente un Dumas arabisé, méconnaissable.

2. Jean-Claude Pirotte, Il est minuit depuis toujours, 1993, p. 131.

3. Didier Daeninckx, Play-back, 1992, p. 21

4. Le Nouvel Observateur $n^{\circ} 1508$, du 30 septembre au 6 octobre 1993, p. 40 à 41. Dessin de Wiaz.

5. Un catalogue déjà fort imposant en a été dressé par Claude Schopp à la fin de son édition des Mémoires.

6. Cf. Charles Grivel, Alexandre Dumas, "le Parler noir". Dans Littérature populaire. Peuple, nation, région. Trames, Publications de l'Université de Limoges, 1987, 205 à 231.

7. D'un article consacré à Marilhat, reproduit dans son Voyage en Egypte, présentation et notes de Paolo Tortonese, La Boîte à Documents, 1991, p. 106. Gautier fait erreur, créole ne veut pas dire « noir » mais « des îles»

8. Cf. Mon Plaisir... en littérature, Gallimard, 1967, p. 104 à 105.

9. Humeurs et humour d'Alexandre Dumas, Grancher, 1993, couverture 4 et p. 13.

10. Alexandre Dumas le Grand. Suivi de "Jacques Bonhomme ", un inédit, Julliard, 1993, p. 143 à 144. Daniel Zimmermann multiplie les indices de même nature : Dumas veut plaire et même conquérir son « beau » sous-chef de bureau, Dumas n'aime que les hommes «beaux », la " beauté » ambiguë de Dumas plaît aux représentants des deux sexes, Dumas joue volontiers de son charme " androgyne ", etc.

11. Chapitre CXXXVIII.

12. Reproduit dans Alexandre Dumas en images, Nilsson, s.d., p. 36.

13. Cité par Gabriel Ferry, Les Dernières années d'Alexandre Dumas, 1867-1870, Calmann Lévy, 1883, p. 25 à 26.

14. Portraits littéraires, Tome 2, 1836, p. 143 à 163.

15. Repris dans Histoire de l'art dramatique en France depuis vingt-cinq ans, Hetzel, 1858, I, p. 84 à 86.

16. Le Soleil Alexandre Dumas, Dentu, 1855. p. 7, 34, 39, 40, 41, 56 et 57. Pour un aperçu, d'ailleurs fort tendancieux, sur l'affaire, cf. D. Zimmermann, op.cit. p. 513 à 517. 
17. Portraits contemporains. Nouvelle édition revue et corrigée. Tome II, 1855, p. 329 à 330 . Tome I, p. 487 à 496 et 71 à 72 .

18. Seuils. Edition du Seuil, 1987, p. 47.

19. Publié en traduction chez Lattès cette année.

20. Version française publiée en 1993 chez Grasset.

21. Eco indique en parallèle que Mussolini donna lui aussi dans le roman-feuilleton, en 1910, et que son livre doit être compris comme une resucée du bon vieux roman populaire façon XIX ${ }^{\mathrm{e}}$. Lire, comme il fallait s'y attendre, tire la conclusion sans ambages : «La littérature populaire et l'idéologie fasciste ont la même origine» [n de novembre 1993, p. 73]. Je proteste.

22. L'expression est de Dumas, $\mathrm{cf}$. $\mathrm{Cl}$. Schopp, Frères d'armes de la révolution romantique. Lettres d'Alexandre Dumas au Baron Taylor et à Adrien Dauzats, Fondation Taylor, 1993, p. 137. 